\title{
Syntheses, Characterization and Biological Evaluation of a Series of 2-Phenylamino-5-(2-Chlorophenyl)- 1,3,4-0xadiazole Derivatives
}

\author{
S. Kumar ${ }^{1}$ and P. K. Srivastava ${ }^{2}$ \\ ${ }^{1}$ Department of Chemistry, Faculty of Science, Iswar Saran P G College, University of Allahabad \\ Allahabad-211004, India \\ ${ }^{2}$ Department of Zoology, Faculty of Science, University of Allahabad, Allahabad-211002, India
}

(Received: April 23, 2018; Accepted: April 18, 2019; Published (Web) June 30, 2019)

\begin{abstract}
Electrochemical synthesis of 2-phenylamino-5-(2-chlorophenyl)-1,3,4-oxadiazoles have been carried out in good yields at platinum electrode through the electrochemical oxidation of acyl thiosemicarbazide at room temperature in acetic acid. Two platinum electrodes in the form of square plates were used as working as well as counter electrode and saturated calomel electrode was used as reference electrode. The structure of the compounds was confirmed by IR, NMR, mass spectral and elemental analyses. The antibacterial activity of the derivatives was also assessed and compared with data against a series of Gram-positive Klebsiella pneumoniae, Escherichia coli and Gramnegative bacteria Streptococcus aureus and Bascillus subtilis. The antifungal activity was assessed against the fungal strain Aspergillus niger, Crysosporium pannical, Pellicularia solmanicolor and Candida albicans and compared against the standard antifungal drug Griesvofulvin.
\end{abstract}

Key words: 1,3,4-Oxadiazoles, Arylthiosemicarbazide, Controlled potential electrolysis, Platinum electrode, Green chemistry.

\section{INTRODUCTION}

Various 1,3,4-oxadiazole derivatives were reported in the literature to have a broad spectrum of biological activities including such as antibacterial ${ }^{1,2}$, anti-HIV $^{1}$, antifungal ${ }^{3,6}$, genotoxic ${ }^{3,6}$, antitubercular ${ }^{6}$, virucidal $^{7}$, antimalarial ${ }^{8}$, insecticidal ${ }^{9}$, herbicidal ${ }^{10}$, analgesic $^{11}$, anti-inflammatory ${ }^{12}$, muscle relaxants $^{13,14}$, anticonvulsant ${ }^{15}$, sedative ${ }^{16}$, hypnotic ${ }^{16}$, anticancer ${ }^{17}$ and lipid peroxidation inhibitors. In the present investigation, a series of substituted 1,3,4oxadiazole derivatives were synthesized as potential antibacterial and antifungal agents by the electrooxidative synthesis.

The oxadiazole ring systems have a long history of application in pharmaceutical and agrochemical industries due to their activity. On the study of literature it have been found that the methods for

Correspondence to: S. Kumar

E-mail: sanjivks77@gmail.com

Dhaka Univ. J. Pharm. Sci. 18(1): 75-83, 2019 (June) DOI: https://doi.org/10.3329/dujps.v18i1.41894 synthesis of oxadiazoles $^{19}$ 2,5-disubstituted or 2amino-5-substituted-1,3,4-oxadiazoles include bromine oxidation of semicarbazide derivative and the cyclodesulfurisation of acylthiosemicarbazide derivative in the solution using $\mathrm{I}_{2} / \mathrm{NaOH}$ or 1,3 -dicyclohexylcarbodimide (DCC) as well as mercury (II) acetate $\left[\mathrm{Hg}(\mathrm{OAc})_{2}\right]$ or yellow mercury (II) oxide $\mathrm{HgO}^{20-22}$ All these methods are usually carried out in various different synthetic steps and requires the heating at high temperature. The handling of these reagents is not only difficult but also very hazardous to the environment. The each stage of the reaction including extraction and purification of the products from the mixture required great precautions. Karen Evans ${ }^{23}$ have synthesized a similar cyclized product by the chemical method in which 2-amino-5substituted-1,3,4-oxadiazoles were prepared by rapid parallel synthesis in efficient one-pot preparation using resin-bound reagents. 
The electrochemical oxidation reaction has several merits. These reactions do not require oxidizing reagents and can be performed at room temperature. Application of electricity as a non conventional energy source for activation of reactants in suitable solvents has now gained popularity over the usual homogeneous and heterogeneous reactions. It provides chemical processes with special attributes, such as enhanced reaction rate, higher yield of pure products, better selectivity and several ecofriendly advantages.

During hit to lead efforts following a recent high throughput screening campaign, we initiated the electrolysis of semicarbazone for the synthesis of 2-amino-5-substituted-1,3,4-oxadiazoles at the platinum electrode as a green synthesis. Later on, it have been found out from the literature that the activity of the oxadiazoles can be enhanced if the amino group is also substituted by an alkyl, aryl or acyl group. Keeping this observation in view and in continuation of our research on the synthesis of heterocyclic compounds containing nitrogen and oxygen with expected biological activity, this paper presents the synthesis of several derivatives of 2phenylamino-5-(2-chlorophenyl)-1,3,4-oxadiazoles which contain phenyl moiety and the study of their antibacterial and antifungal activity.

\section{EXPERIMENTAL}

Physical measurements. Melting points were determined using open capillary tubes and were uncorrected. The purity of the synthesized compounds were ascertained by thin layer chromatography on Silica gel $\mathrm{G} 60 \mathrm{~F}_{254}$ plates from Merck and visualized by exposure to iodine vapor. Spectra were obtained as follows: Infra red (IR) spectra were recorded on a Shimadzu 8201 PC IR spectrophotometer $\left(4000-400 \mathrm{~cm}^{-1}\right)$ in $\mathrm{KBr}$ pellets and reported in $\mathrm{cm}^{-1}$. ${ }^{1} \mathrm{H} \mathrm{NMR}$ and ${ }^{13} \mathrm{C}$ NMR spectra were measured at room temperature on Bruker DRX $300 \mathrm{MHz}$ and $75 \mathrm{MHz}$ FT spectrometer instruments, with tetramethylsilane (TMS) and $\mathrm{CDCl}_{3}$ or $\mathrm{C}_{6} \mathrm{D}_{6}$ and their chemical shifts are reported in $\delta$ (parts per million). Carbon multiplities were assigned by DEPT techniques. Microanalysis for $\mathrm{C}, \mathrm{H}, \mathrm{N}$ was performed in the Elementar Vario EL III.

General procedures for the synthesis of acylthiosemicarbazide (4a-4l). Arylisothiocyanate 3 was prepared directly from an arylamine. The sparingly soluble ammonium aryldiathiacarbamate was obtained by the reaction of an arylamine, $\mathrm{CS}_{2}$, and aqueous ammonia. Then aryldiathiacarbamate is decomposed by lead nitrate to produce arylisothiocyanate. The equimolar amount of arylhydrazine $\mathbf{2}$ and arylisothiocynate $\mathbf{3}$ were mixed in a small beaker with continuous stirring. After few minutes of stirring, the mixture was left overnight, which gave a solid compound arylthiosemicarbazide 4.

(4a): IR/ $\mathrm{cm}^{-1}: 1015(\mathrm{~N}-\mathrm{N}), 1265(\mathrm{C}=\mathrm{S}), 1449$ $(\mathrm{C}=\mathrm{N}+\mathrm{ArC}=\mathrm{C}), 1632(\mathrm{C}=\mathrm{O}), 3068(\mathrm{ArC}-\mathrm{H}) ;{ }^{1} \mathrm{H}-$ NMR: $\delta$ 6.88-7.98 (m, 8H, ArH), 10.60-11.96 (s, 3H, $\mathrm{NH}) ;{ }^{13} \mathrm{C}-\mathrm{NMR}: \delta 116.1,119,126.4,127.6,128.6$, $129.8,133.4,140.7,143.06,148.1,159.5,181.6$; $\mathrm{M} / \mathrm{Z}=305.5\left[\mathrm{M}^{+}\right]$. Calcd. for $\mathrm{C}_{14} \mathrm{H}_{12} \mathrm{~N}_{3} \mathrm{OSCl}$ : C, 54.98; H, 3.92; N, 13.74; Cl, 11.62; S, 10.47. Found: C, 54.09; H, 3.61; N, 13.46; Cl, 11.22; S, 10.17 .

General procedure for the synthesis of $2-\mathrm{N}$ phenylamino-5-(2-chlorophenyl)-1,3,4-oxadiazol (1a-l). Arylthiosemicarbazide 4, 4a (1000 mg, 3.27 mmol) and $\mathrm{LiClO}_{4}(106 \mathrm{mg}, 0.67 \mathrm{mmol})$ were dissolved in acetic acid $(100 \mathrm{ml})$ to prepare the reaction mixture for electrolysis.

Electrolysis. Preparative scale controlled potential electrolyses $^{21-29}$ were performed at room temperature in $250 \mathrm{ml}$ three-electrode cell assembly with platinum plate $(1.0 \mathrm{~cm} \times 1.0 \mathrm{~cm})$ as working as well as counter electrode and saturated calomel electrode (SCE) as reference electrode. Magnetic stirrer was used for the proper mixing of reaction mixture.

All the electrolysis experiments were carried out at their corresponding oxidation potentials and were completed in 3 to $5 \mathrm{hrs}$. After which no oxidation product was seen to diffuse in the bulk. All the products were solid and colored and entirely different from the starting compound. The current potential data was recorded with the help of a potentiostat at 
the interval of $15 \mathrm{~min}$ as depicted in table 1 . Approximately $4.5-6 \mathrm{Fmol}^{-1}$ quantity of electricity was passed for the electrolysis which is very small in comparison to energy used in other conventional methods.

Extraction. The products were extracted from the acetic acid solution to chloroform layer after diluting reaction solution with double distilled water by the simple solvent extraction technique. Twoimmiscible layers of acetic acid containing water and chloroform were shaken in a seperatory funnel and allowed to settle down. After some time the chloroform layer containing the product oxadiazole, was removed. The extracted chloroform layer was evaporated with the help of rotatory evaporator and collected while the product oxadiazole $\mathbf{1}$ remain in flask, were obtained in the excellent yield. The purity of the compounds oxadiazoles have been checked by the TLC.

2-Phenylamino-5-(2-chlorophenyl)-1,3,4-oxadiazole (1a). Brown crystals; m.p. $152-154{ }^{0} \mathrm{C}$; $\mathrm{IR} / \mathrm{cm}^{-1}$ : $3230(\mathrm{NH}), 3045(\mathrm{ArC}-\mathrm{H}), 1607(\mathrm{C}=\mathrm{N})$, 1265, 1069 (C-O-C), 910, 860, 735 (benzene), 600800 ( $\mathrm{Ar}-\mathrm{Cl}) ;{ }^{1} \mathrm{H}$ NMR (DMSO-d ${ }_{6}, \delta \mathrm{ppm}$ ): 10.25 $(1 \mathrm{H}, \mathrm{s}, \mathrm{NH}), 7.29-7.47$ (9H, m, ArH); ${ }^{13} \mathrm{C} \mathrm{NMR}$ (DMSO-d $\left.{ }_{6}, \delta \mathrm{ppm}\right): 176.2,159.8,148.1,140.8$, $129.8,129,128.6,127.9,126.4,119,116.1 ; \mathrm{m} / z=$ $272\left(\mathrm{M}^{+}+1\right)$. Calcd. for $\mathrm{C}_{14} \mathrm{H}_{10} \mathrm{~N}_{3} \mathrm{OCl}: \mathrm{C}, 61.89 ; \mathrm{H}$, 3.70; N, 15.46; Cl, 13.04. Found: C, 61.23; H, 3.54; $\mathrm{N}, 15.02 ; \mathrm{Cl}, 12.89$.

2-Phenylamino-5-(2-hydroxyphenyl)-1,3,4oxadiazole (1b). Yellow crystals; m.p. $144-146{ }^{\circ} \mathrm{C}$; $\mathrm{IR} / \mathrm{cm}^{-1}$ : $3440(\mathrm{O}-\mathrm{H}), 3250(\mathrm{NH}), 3045(\mathrm{ArC}-\mathrm{H})$, $1607(\mathrm{C}=\mathrm{N}), 1265,1069$ (C-O-C), 910, 860, 735 (benzene); ${ }^{1} \mathrm{H}$ NMR (DMSO-d ${ }_{6}, \delta$ ppm): $11.69(1 \mathrm{H}$, s, OH), $10.35(1 \mathrm{H}, \mathrm{s}, \mathrm{NH}), 7.28-7.99(9 \mathrm{H}, \mathrm{m}, \mathrm{ArH})$; ${ }^{13} \mathrm{C}$ NMR (DMSO-d $6, \delta$ ppm): 175.2, 159.6, 153.3, $148.4,128.8,128.3,127.90,121.1,119.12,116.2$, 115.7; $\mathrm{m} / z=254\left(\mathrm{M}^{+}+1\right)$. Calcd. for $\mathrm{C}_{14} \mathrm{H}_{11} \mathrm{~N}_{3} \mathrm{O}_{2}: \mathrm{C}$, 66.40; H, 4.38; N, 16.59. Found: C, 65.85; H, 4.07; $\mathrm{N}, 16.23$.

2-Phenylamino-5-(2-nitrophenyl)-1,3,4-oxadiazole (1c). Brown needles; m.p. $172-174{ }^{0} \mathrm{C} ; \mathrm{IR} / \mathrm{cm}^{-1}$ : $3260(\mathrm{NH}), 3055(\mathrm{ArC}-\mathrm{H}), 1604(\mathrm{C}=\mathrm{N}), 1580(\mathrm{Ar}-$
$\mathrm{NO}_{2}$ ), 1255, 1080 (C-O-C), 950, 860, 740 (benzene); ${ }^{1} \mathrm{H}$ NMR (DMSO-d $\left.\mathrm{d}_{6}, \delta \mathrm{ppm}\right): 10.32(1 \mathrm{H}, \mathrm{s}, \mathrm{NH})$, 6.97-7.88 (9H, m, ArH); ${ }^{13} \mathrm{C}$ NMR (DMSO-d ${ }_{6}, \delta$ ppm): 174.4, 157.4, 148.4, 146.3, 135.3, 134.4, $128.8,127.8,123.1,119.12,116.2 ; \mathrm{m} / \mathrm{z}=283\left(\mathrm{M}^{+}+\right.$ 1). Calcd. for $\mathrm{C}_{14} \mathrm{H}_{10} \mathrm{~N}_{4} \mathrm{O}_{3}: \mathrm{C}, 59.57 ; \mathrm{H}, 3.54 ; \mathrm{N}$, 19.85. Found: C, 58.92; H, 3.46; N, 18.63 .

2-(2-Methoxyphenyl)amino-5-(2-chloro-phenyl) 1,3,4-oxadiazole (1d). Dark brownish needles; m.p. 186-188 ${ }^{0} \mathrm{C}$; IR/cm ${ }^{-1}: 3244(\mathrm{NH}), 2822\left(\mathrm{O}-\mathrm{CH}_{3}\right)$, 2927 (ArC-H), 2853 (alipC-H), $1611(\mathrm{C}=\mathrm{N}), 1250$, 1062 (C-O-C), 915, 870, 675 (benzene), 600-800 (Ar-Cl); ${ }^{1} \mathrm{H}$ NMR (DMSO-d ${ }_{6}, \delta$ ppm): $10.35(1 \mathrm{H}, \mathrm{s}$, $\mathrm{NH}), 6.92-7.45(8 \mathrm{H}, \mathrm{m}, \mathrm{ArH}), 3.74\left(3 \mathrm{H}, \mathrm{s}, \mathrm{OCH}_{3}\right)$; ${ }^{13} \mathrm{C}$ NMR (DMSO-d $\mathrm{d}_{6}, \delta \mathrm{ppm}$ ): 174.7, 158.4, 147.5, $140.8,133.5,128.6,127.9,126.4,122.1,119.9$, 117.1, 115.4, 53.7; $\mathrm{m} / \mathrm{z}=304\left(\mathrm{M}^{+}+1\right)$. Calcd. for $\mathrm{C}_{15} \mathrm{H}_{13} \mathrm{~N}_{3} \mathrm{O}_{2} \mathrm{Cl}: \mathrm{C}, 59.60 ; \mathrm{H}, 4.30 ; \mathrm{N}, 13.90 ; \mathrm{Cl}$, 11.75. Found: C, 58.26; H, 4.22; N, 13.15; Cl, 11.29.

2-(2-Methoxyphenyl)amino-5-(4-N,N-diethylaminophenyl)-1,3,4-oxadiazole (1e). Dark brown crystals; m.p. 191-193 ${ }^{0} \mathrm{C}$; IR/ $/ \mathrm{cm}^{-1}$ : $3261(\mathrm{NH}), 3045$ (ArC-H), $2815\left(\mathrm{O}^{\left.-\mathrm{CH}_{3}\right),} 2855\right.$ (alipC-H), 1609 $(\mathrm{C}=\mathrm{N}), \quad 1270, \quad 1069 \quad$ (C-O-C), 915, 870, 790 (benzene); ${ }^{1} \mathrm{H}$ NMR (DMSO-d 6 , $\delta$ ppm): $10.48(1 \mathrm{H}$, s, NH), $8.03(2 \mathrm{H}, \mathrm{d}, J=8.3 \mathrm{~Hz}, \mathrm{ArH}), 6.93-7.46(4 \mathrm{H}$, $\mathrm{m}, \operatorname{ArH}), 6.91(2 \mathrm{H}, \mathrm{d}, J=8.3 \mathrm{~Hz}, \operatorname{ArH}), 6.49-7.75$ $(8 \mathrm{H}, \mathrm{m}, \mathrm{ArH}), 4.07\left(2 \mathrm{H}, \mathrm{q}, J=7.3 \mathrm{~Hz}, \mathrm{CH}_{3} \mathrm{CH}_{2} \mathrm{~N}\right)$, $3.11\left(3 \mathrm{H}, \quad \mathrm{s}, \mathrm{OCH}_{3}\right), 1.33(3 \mathrm{H}, \mathrm{t}, \quad J=7.3 \mathrm{~Hz}$, $\mathrm{CH}_{3} \mathrm{CH}_{2} \mathrm{~N}$ ); ${ }^{13} \mathrm{C}$ NMR (DMSO-d 6 , $\delta \mathrm{ppm}$ ): 175.2, $159.8,147.5,142.4,133.5,127.7,122.3,119.4$, 117.6, 115.1, 53.8, 35.5, 17.9; $\mathrm{m} / \mathrm{z}=339\left(\mathrm{M}^{+}+1\right)$. Calcd. for $\mathrm{C}_{19} \mathrm{H}_{22} \mathrm{~N}_{4} \mathrm{O}_{2}: \mathrm{C}, 67.43 ; \mathrm{H}, 6.55 ; \mathrm{N}, 16.56$. Found: C, 66.87; H, 6.42; N, 16.25 .

2-(2-Methoxyphenyl)amino-5-(2-nitrophenyl)1,3,4-oxadiazole (1f). Dark brown crystals; m.p. 195$197{ }^{0} \mathrm{C}$; IR $/ \mathrm{cm}^{-1}$ : $3260(\mathrm{NH}), 2860$ (alipC-H), 2822 $\left(\mathrm{O}-\mathrm{CH}_{3}\right), 1585\left(\mathrm{Ar}-\mathrm{NO}_{2}\right), 1608(\mathrm{C}=\mathrm{N}), 1280,1066$ (C-O-C), 925, 890, 785 (benzene); ${ }^{1} \mathrm{H}$ NMR (DMSO$\left.\mathrm{d}_{6}, \delta \mathrm{ppm}\right): 10.29(1 \mathrm{H}, \mathrm{s}, \mathrm{NH}), 6.89-7.75(8 \mathrm{H}, \mathrm{m}$, $\mathrm{ArH}), 3.78\left(3 \mathrm{H}, \mathrm{s}, \mathrm{OCH}_{3}\right) ;{ }^{13} \mathrm{C}$ NMR $\left(\mathrm{DMSO}_{6}, \delta\right.$ ppm): 174.4, 157.4, 147.5, 146.2, 135.3, 134.4, 133.5, 127.8, 123.1, 122.1, 119.9, 117.1, 115.4, 53.8; $m / z=313\left(\mathrm{M}^{+}+1\right)$. Calcd. for $\mathrm{C}_{15} \mathrm{H}_{12} \mathrm{~N}_{4} \mathrm{O}_{4}: \mathrm{C}, 57.70$; 
H, 3.85; N, 17.94. Found: C, 57.16; H, 3.72; N, 17.15 .

2-(4-Methoxyphenyl)amino-5-(2-chloro-phenyl)-1,3,4-oxadiazole (1g). Yellow crystals; m.p. 99$101{ }^{0} \mathrm{C}$; IR/ $\mathrm{cm}^{-1}$ : $3236(\mathrm{NH}), 3033(=\mathrm{C}-\mathrm{H}), 3030$ (ArC-H), 2855 (aliphC-H), $2820\left(\mathrm{O}^{\left.-\mathrm{CH}_{3}\right),} 1670\right.$ $(\mathrm{C}=\mathrm{C}), 1606(\mathrm{C}=\mathrm{N}), 1262,1067$ (C-O-C), 920, 860, 790 (benzene), 600-800 (Ar-Cl); ${ }^{1} \mathrm{H}$ NMR (DMSO$\left.\mathrm{d}_{6}, \delta \mathrm{ppm}\right): 10.32(1 \mathrm{H}, \mathrm{s}, \mathrm{NH}), 8.03(2 \mathrm{H}, \mathrm{d}, J=8.5 \mathrm{~Hz}$, $\mathrm{ArH}), 7.35-7.45$ (4H, m, ArH), 6.98 (2H, d, $J=8.3$ $\mathrm{Hz}, \mathrm{ArH}), 3.77\left(3 \mathrm{H}, \mathrm{s}, \mathrm{OCH}_{3}\right) ;{ }^{13} \mathrm{C}$ NMR (DMSO-d 6 , $\delta \mathrm{ppm}): 174.4,157.4,141.3,133.5,132.6,128.6$, $127.8,126.2,119.5,114.1,55.5 ; \mathrm{m} / z=304\left(\mathrm{M}^{+}+1\right)$. Calcd. for $\mathrm{C}_{15} \mathrm{H}_{13} \mathrm{~N}_{3} \mathrm{O}_{2} \mathrm{Cl}: \mathrm{C}, 59.47 ; \mathrm{H}, 4.30 ; \mathrm{N}$, 13.87; Cl, 11.73. Found: C, 58.89; H, 4.03; N, 13.22; $\mathrm{Cl}, 11.36$.

2-(4-Methoxyphenyl)amino-5-(2-nitrophenyl)1,3,4-oxadiazole (1h). Brownish needles; m.p. 172$174{ }^{0} \mathrm{C} ; \mathrm{IR} / \mathrm{cm}^{-1}: 3244(\mathrm{NH}), 2870$ (alipC-H), 2822 $\left(\mathrm{O}-\mathrm{CH}_{3}\right), 2927(\mathrm{ArC}-\mathrm{H}), 1611(\mathrm{C}=\mathrm{N}), 1583(\mathrm{Ar}-$ $\mathrm{NO}_{2}$ ), 1250, 1062 (C-O-C), 915, 870, 675 (benzene); ${ }^{1} \mathrm{H}$ NMR (DMSO-d $\mathrm{d}_{6}, \delta \mathrm{ppm}$ ): 10.35 (1H, s, NH), 8.03 $(2 \mathrm{H}, \mathrm{d}, J=8.5 \mathrm{~Hz}, \mathrm{ArH}), 7.31-7.51(4 \mathrm{H}, \mathrm{m}, \mathrm{ArH})$, $6.98(2 \mathrm{H}, \mathrm{d}, J=8.3 \mathrm{~Hz}, \mathrm{ArH}), 3.74\left(3 \mathrm{H}, \mathrm{s}, \mathrm{OCH}_{3}\right) ;{ }^{13} \mathrm{C}$ NMR (DMSO-d $\mathrm{d}_{6}, \delta$ ppm): 174.7, 158.4, 146.3, 135.5, $134.8,132.5,127.6,123.2,114.1,53.8 ; \mathrm{m} / \mathrm{z}=313$ $\left(\mathrm{M}^{+}+1\right)$. Calcd. for $\mathrm{C}_{15} \mathrm{H}_{12} \mathrm{~N}_{4} \mathrm{O}_{4}: \mathrm{C}, 57.69 ; \mathrm{H}, 3.84 ; \mathrm{N}$, 17.94. Found: C, 57.11; H, 3.76; N, 17.25.

2-(4-Methoxyphenyl)amino-5-(2,4-dinitrophenyl)-1,3,4-oxadiazole (1i). Brownish needles; m.p. 182-184 ${ }^{0} \mathrm{C}$; IR/ $/ \mathrm{cm}^{-1}$ : $3244(\mathrm{NH}), 2870$ (alipC-H),

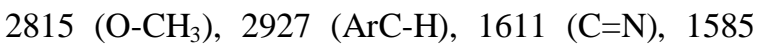
$\left(\mathrm{Ar}-\mathrm{NO}_{2}\right)$, 1250, 1062 (C-O-C), 915, 870, 675 (benzene); ${ }^{1} \mathrm{H}$ NMR (DMSO-d $\left.\mathrm{d}_{6}, \delta \mathrm{ppm}\right): 10.35(1 \mathrm{H}$, s, NH), $8.78(1 \mathrm{H}, \mathrm{s}, \operatorname{ArH}), 8.52(1 \mathrm{H}, \mathrm{d}, J=8.7 \mathrm{~Hz}$, ArH), $8.03(1 \mathrm{H}, \mathrm{d}, J=8.5 \mathrm{~Hz}, \operatorname{ArH}), 7.69(1 \mathrm{H}, \mathrm{d}$, $J=8.7 \mathrm{~Hz}, \mathrm{ArH}), 6.98(2 \mathrm{H}, \mathrm{d}, J=8.3 \mathrm{~Hz}, \mathrm{ArH}), 3.14$ $\left(3 \mathrm{H}, \quad \mathrm{s}, \quad \mathrm{OCH}_{3}\right) ;{ }^{13} \mathrm{C}$ NMR (DMSO-d $\left.\mathrm{d}_{6}, \delta \mathrm{ppm}\right)$ : $174.75,158.47,147.2,141.3,136.0,129.4,128.8$, $127.8,126.8,125.8,117.9,53.8 ; \mathrm{m} / \mathrm{z}=268\left(\mathrm{M}^{+}+1\right)$. Calcd. for $\mathrm{C}_{15} \mathrm{H}_{11} \mathrm{~N}_{5} \mathrm{O}_{6}: \mathrm{C}, 67.41 ; \mathrm{H}, 4.12 ; \mathrm{N}, 26.25$. Found: C, 66.76; H, 4.01; N, 25.96.

2-(4-Methylphenyl)amino-5-(2-chlorophenyl)1,3,4-oxadiazole (1j). Brownish needles; m.p 189-
$191{ }^{0} \mathrm{C}$; IR/: 3244 (NH), 2870 (alipC-H), 2927 (ArCH), $1611(\mathrm{C}=\mathrm{N})$, 1250, 1062 (C-O-C), 915, 870, 675 (benzene), 600-800 (ArCl); ${ }^{1} \mathrm{H}$ NMR (DMSO-d $6, \delta$ ppm): $10.36(1 \mathrm{H}, \mathrm{s}, \mathrm{NH}), 8.03(2 \mathrm{H}, \mathrm{d}, J=8.3 \mathrm{~Hz}$, $\mathrm{ArH}), 7.30-7.45(4 \mathrm{H}, \mathrm{m}, \mathrm{ArH}), 7.01(2 \mathrm{H}, \mathrm{d}, J=8.3$ $\mathrm{Hz}, \mathrm{ArH}), 1.12\left(3 \mathrm{H}, \mathrm{s}, \mathrm{CH}_{3}\right) ;{ }^{13} \mathrm{C}$ NMR (DMSO-d 6 , $\delta$ ppm): 174.7, 158.4, 141.3, 140.8, 136.1, 133.3, 129.3, 128.6, 127.6, 126.8, 126.4, 125.8, 21.18; $m / z=343\left(\mathrm{M}^{+}+1\right)$. Calcd. for $\mathrm{C}_{15} \mathrm{H}_{12} \mathrm{~N}_{3} \mathrm{OCl}: \mathrm{C}, 63.04$; $\mathrm{H}, 4.20 ; \mathrm{N}, 14.71 ; \mathrm{Cl}$ 12.43. Found: C, 62.68; H, $4.05 ; \mathrm{N}, 13.92 ; \mathrm{Cl} 12.23$.

2-(4-Methylphenyl)amino-5-(4-N,N-diethylaminophenyl)-1,3,4-oxadiazole (1k). Yellow crystals; m.p. $190-192^{0} \mathrm{C}$; IR/cm ${ }^{-1}$ : $3261(\mathrm{NH}), 3045(\mathrm{ArC}-\mathrm{H})$, 2855 (alipC-H), $3033(=\mathrm{C}-\mathrm{H}), 1609(\mathrm{C}=\mathrm{N}), 1270$, 1069 (C-O-C), 915, 870, 790 (benzene); ${ }^{1} \mathrm{H}$ NMR (DMSO-d $\left.\mathrm{d}_{6}, \delta \mathrm{ppm}\right): 10.55(1 \mathrm{H}, \mathrm{s}, \mathrm{NH}), 8.03(2 \mathrm{H}, \mathrm{d}$, $J=8.3 \mathrm{~Hz}, \operatorname{ArH}), 7.25(2 \mathrm{H}, \mathrm{d}, J=8.3 \mathrm{~Hz}, \operatorname{ArH}), 6.93-$ $7.46(4 \mathrm{H}, \mathrm{m}, \mathrm{ArH}), 4.05(2 \mathrm{H}, \mathrm{q}, J=7.3 \mathrm{~Hz}$, $\left.\mathrm{CH}_{3} \mathrm{CH}_{2} \mathrm{~N}\right), 1.12\left(3 \mathrm{H}, \mathrm{s}, \mathrm{CH}_{3}\right), 1.35(3 \mathrm{H}, \mathrm{t}, J=7.3 \mathrm{~Hz}$, $\mathrm{CH}_{3} \mathrm{CH}_{2} \mathrm{~N}$ ); ${ }^{13} \mathrm{C}$ NMR (DMSO-d $6 . \delta$ ppm): 174.7, 158.4, 142.4, 141.5, 136, 129.3, 127.7, 126.8, 125.8, 119.4, 35.9, 21.18, 17.7; $\mathrm{m} / \mathrm{z}=323\left(\mathrm{M}^{+}+1\right)$. Calcd. for $\mathrm{C}_{19} \mathrm{H}_{22} \mathrm{~N}_{4} \mathrm{O}: \mathrm{C}, 78.26 ; \mathrm{H}, 6.83 ; \mathrm{N}, 17.39$. Found: C, 77.87; H, 6.42; N, 17.27.

\section{2-(4-Methylphenyl)amino-5-(2,4-dinitrophe-} nyl)-1,3,4-oxadiazole (11). Brownish needles; m.p. 183-185 ${ }^{0} \mathrm{C}$; IR/cm ${ }^{-1}: 3244(\mathrm{NH}), 2870$ (alipC-H), $2927(\mathrm{ArC}-\mathrm{H}), 1611(\mathrm{C}=\mathrm{N}), 1585\left(\mathrm{Ar}-\mathrm{NO}_{2}\right), 1250$, 1062 (C-O-C), 915, 870, 675 (benzene); ${ }^{1} \mathrm{H}$ NMR (DMSO-d $\left.\mathrm{d}_{6}, \delta \mathrm{ppm}\right): 10.35(1 \mathrm{H}, \mathrm{s}, \mathrm{NH}), 8.78(1 \mathrm{H}, \mathrm{s}$, ArH), $8.52(1 \mathrm{H}, \mathrm{d}, J=8.7 \mathrm{~Hz}, \operatorname{ArH}), 7.69(1 \mathrm{H}, \mathrm{d}$, $J=8.5 \mathrm{~Hz}, \mathrm{ArH}), 7.33(2 \mathrm{H}, \mathrm{d}, J=8.3 \mathrm{~Hz}, \mathrm{ArH}), 7.26$ $(2 \mathrm{H}, \mathrm{d}, J=8.3 \mathrm{~Hz}, \mathrm{ArH}), 1.12\left(3 \mathrm{H}, \mathrm{s}, \mathrm{CH}_{3}\right) ;{ }^{13} \mathrm{C} \mathrm{NMR}$ (DMSO-d $\mathrm{d}_{6}, \delta \mathrm{ppm}$ ): 174.7, 158.4, 153.3, 141.3, $136.1,129.3,128.5,127.9,126.8,125.8,119.4$, 115.7, 21.18; $\mathrm{m} / \mathrm{z}=342\left(\mathrm{M}^{+}+1\right)$. Calcd. for $\mathrm{C}_{15} \mathrm{H}_{11} \mathrm{~N}_{5} \mathrm{O}_{5}: \mathrm{C}, 52.78 ; \mathrm{H}, 3.22 ; \mathrm{N}, 20.53$. Found: $\mathrm{C}$, 52.06; H, 3.12; N, 20.25.

Antimicrobial activity. All the title compounds were tested for their antibacterial and antifungal activities by utilizing the disc diffusion method. ${ }^{30,31}$ Whatman No.1 filter paper discs of $6 \mathrm{~mm}$ diameter, placed in a petri dish, were autoclaved. The test 
compounds in measured quantities $(1.0 \mathrm{mg}, 0.5 \mathrm{mg}$ ) were dissolved in $5 \mathrm{ml}$ of dimethylformamide to produce $200 \mathrm{ppm}$ and $100 \mathrm{ppm}$ solutions, respectively. The filter paper discs were allowed to dry and the amount of the substance per disc was taken as 500 and $250 \mu \mathrm{g}$. The bacterial $(24 \mathrm{hrs})$ and fungal $(48 \mathrm{~h})$ cultures from the slants were diluted with sterile water and mixed thoroughly to prepare a clear homogeneous suspension. These suspensions were uniformly spread on solidified agar (nutrient and potato dextrose agar) medium. The filter paper discs prepared from dimethylformamide medium were carefully placed over the spreaded cultures and incubated at $37{ }^{\circ} \mathrm{C}$ for $24 \mathrm{~h}$ for bacteria and at 28 $30^{\circ} \mathrm{C}$ for $48 \mathrm{~h}$ for fungi. Paper discs treated with dimethylformamide alone served as control. After the incubation period the plates were examined for inhibition zones. The diameters of inhibition zones (including the diameter of the disc) were measured. All determinations were made in triplicate for each of the compounds and the average value was taken.

\section{RESULTS AND DISCUSSION}

A novel series of 2-phenylamino-5-(2chlorophenyl)-1,3,4-oxadiazoles (1a-l) have been synthesized in good yields using the synthetic route outlined in scheme 1 and scheme 2. IR, ${ }^{1} \mathrm{H}$ NMR, ${ }^{13} \mathrm{C}$ NMR and mass spectral data are in agreement with the proposed structures of all synthesized compounds.

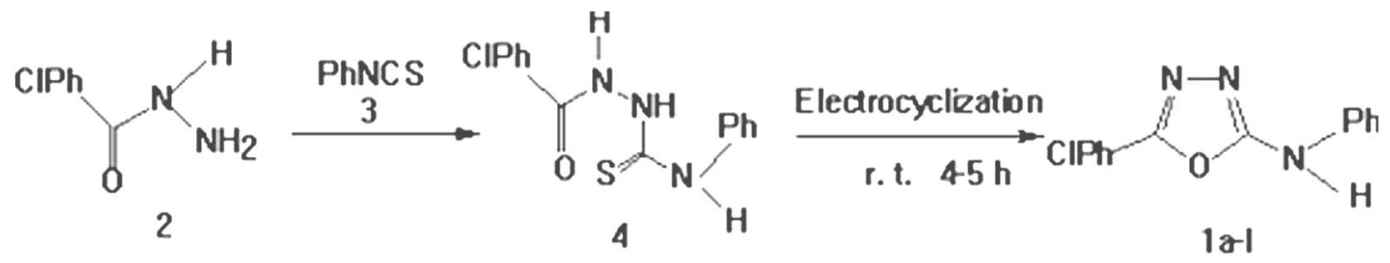

Scheme 1

In the IR spectrum of $\mathbf{2}$ showed broad stretching bands at around 3335 and $3278 \mathrm{~cm}^{-1}$ due to amine/amide $\mathrm{NH}$ while strong stretching band at $1615 \mathrm{~cm}^{-1}$ was attributed to amide carbonyl. ${ }^{1} \mathrm{H}$ NMR spectrum showed a singlet at $\delta 4.51$ and 9.81 which were accounted for $\mathrm{NH}_{2}$ and $\mathrm{NH}$ which disappeared on $\mathrm{D}_{2} \mathrm{O}$ exchange. The four protons of phenyl moiety resonated as multiplets at $\delta 6.68-7.90$. The mass spectrum of 2a showed a molecular ion peak at $\mathrm{m} / \mathrm{z}$ 170.5 which confirmed its molecular weight.

In the IR spectrum of $\mathbf{4}$ displayed broad stretching bands at around $1632 \mathrm{~cm}^{-1}$ for carbonyl and $1265 \mathrm{~cm}^{-1}$ for $\mathrm{C}=\mathrm{S}$ bonding. ${ }^{1} \mathrm{H}$ NMR spectrum showed a singlet at $\delta$ 10.60-11.96 which were accounted for NH. The four protons of phenyl moiety resonated as two doublets at $\delta 7.67$ and 7.89 while five aryl protons show the multiplets at $\delta 6.96$ 7.25. The mass spectrum of $\mathbf{4}$ showed a molecular ion peak at $\mathrm{m} / \mathrm{z} 305.5$ which confirmed its molecular weight.
Lack of ${ }^{1} \mathrm{H}$ NMR resonances observed with $\mathrm{NH}$ and $\mathrm{NH}_{2}$ functions in the ${ }^{1} \mathrm{H}$ NMR spectrum of $\mathbf{1}$ proved that ring closure starting from $\mathbf{4}$ resulted in the formation of 1,3,4-oxadiazole ring. This was further substantiated by the ${ }^{13} \mathrm{C}$ NMR data of $\mathbf{1}$ which showed a peak $\delta 170-173$ and $155-160$ due to $C_{2}$ and $\mathrm{C}_{5}$ of oxadiazole. The IR spectrum showed beaks at $1600-1622 \mathrm{~cm}^{-1}$ for $(\mathrm{C}=\mathrm{N}-\mathrm{N}=\mathrm{C})$ and $1062-1075 \mathrm{~cm}^{-1}$ for $(\mathrm{C}-\mathrm{O}-\mathrm{C})$ in the compounds 1a-l which confirmed the synthesis of 1,3,4-oxadiazoles.

The antibacterial activity of compounds 1a-l was studied against the growth of Klebsilla penumoniae, Escherichia coli, (Gram-negative) and Basillus subtilis, Streptococus aureus (Gram-positive) organisms at the three concentrations (25, 50 and 100 ppm) taking Penicillin as the standard (Table 2). The majority of the compounds exhibited significant (good) antibacterial activity against E. coli, $K$. pneumonia, B. subtilis and $S$. aureus as compared to penicillin. The screening results of antibacterial activity revealed that compound $\mathbf{1 a}, \mathbf{d}, \mathbf{g}$ and $\mathbf{1 j}$ 
exhibited greater activity than the standard Penicillin. Compounds 1c, f, h, i, I display approximately similar or slightly less activity than the standard. The remaining compounds exhibited weak to moderate antibacterial activity against all bacterial strains used for our evaluation.

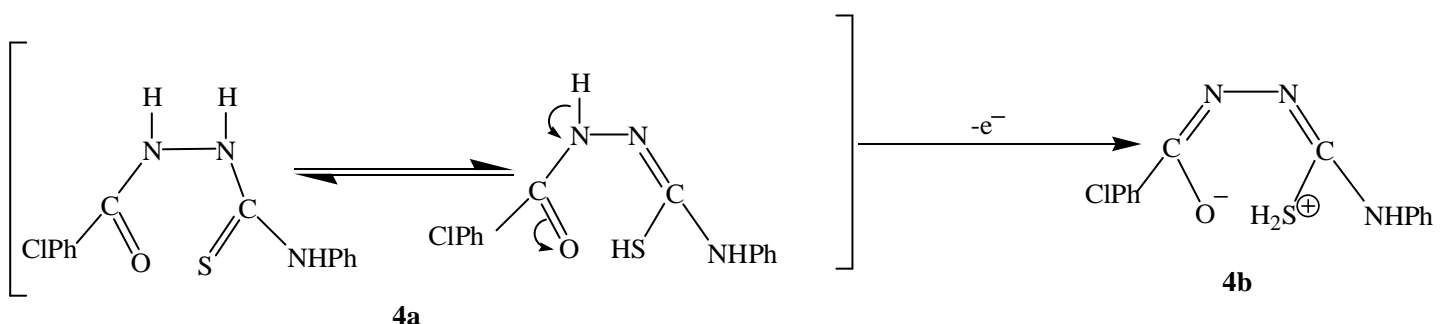

$4 \mathbf{a}$

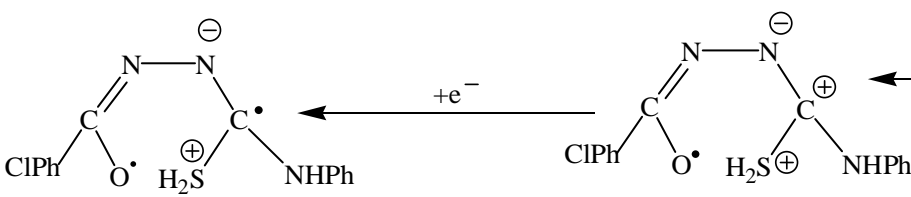

$4 c$<smiles>CC(C)C1=NN(C)C([SiH3])(Nc2ccccc2)O1</smiles>

$4 \mathbf{f}$

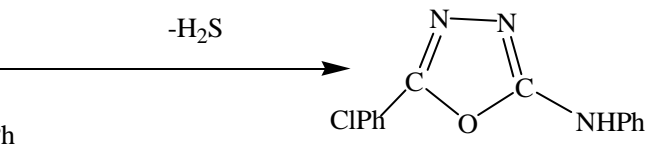

Scheme 2. Mechanistic proposal

Table 1. Current, potential and yield in the synthesis of 1,3,4-oxadiazole derivatives (1a-11).

\begin{tabular}{lllllll}
\hline Compound & $\begin{array}{l}\mathrm{R}^{1} \\
(5-)\end{array}$ & $\begin{array}{l}\mathrm{R}^{2} \\
(2 \text {-amino- })\end{array}$ & Time $[\mathrm{h}]$ & $\begin{array}{l}\text { Applied potential } \\
{[\mathrm{V}]}\end{array}$ & $\begin{array}{l}\text { Current } \\
{[\mathrm{mA}]}\end{array}$ & $\begin{array}{l}\text { Yield } \\
{[\%]}\end{array}$ \\
\hline $\mathbf{1 a}$ & $2-\mathrm{ClC}_{6} \mathrm{H}_{4}$ & $\mathrm{C}_{6} \mathrm{H}_{5}$ & 3 & 1.80 & 1180 & 83 \\
$\mathbf{1 b}$ & $2-(\mathrm{OH}) \mathrm{C}_{6} \mathrm{H}_{4}$ & $\mathrm{C}_{6} \mathrm{H}_{5}$ & 3 & 2.10 & 1016 & 76 \\
$\mathbf{1 c}$ & $2-\left(\mathrm{NO}_{2}\right) \mathrm{C}_{6} \mathrm{H}_{4}$ & $\mathrm{C}_{6} \mathrm{H}_{5}$ & 4 & 1.95 & 1213 & 79 \\
$\mathbf{1 d}$ & $2-\mathrm{ClC}_{6} \mathrm{H}_{4}$ & $2-\mathrm{OMeC}_{6} \mathrm{H}_{4}$ & 5 & 2.00 & 1350 & 71 \\
$\mathbf{1 e}$ & $2-\left(\mathrm{Et}_{2} \mathrm{~N}_{2}\right) \mathrm{C}_{6} \mathrm{H}_{4}$ & $2-\mathrm{OMeC}_{6} \mathrm{H}_{4}$ & 4 & 1.75 & 1055 & 81 \\
$\mathbf{1 f}$ & $2-\left(\mathrm{NO}_{2}\right) \mathrm{C}_{6} \mathrm{H}_{4}$ & $2-\mathrm{OMeC}_{6} \mathrm{H}_{4}$ & 3 & 2.15 & 1115 & 84 \\
$\mathbf{1 g}$ & $2-\mathrm{ClC}_{6} \mathrm{H}_{4}$ & $4-\mathrm{OMeC}_{6} \mathrm{H}_{4}$ & 4 & 1.85 & 1185 & 78 \\
$\mathbf{1 h}$ & $2-\left(\mathrm{NO}_{2}\right) \mathrm{C}_{6} \mathrm{H}_{4}$ & $4-\mathrm{OMeC}_{6} \mathrm{H}_{4}$ & 5 & 1.90 & 1265 & 73 \\
$\mathbf{1 i}$ & $2,4-\left(\mathrm{NO}_{2}\right)_{2} \mathrm{C}_{6} \mathrm{H}_{3}$ & $4-\mathrm{OMeC}_{6} \mathrm{H}_{4}$ & 4 & 2.10 & 1232 & 76 \\
$\mathbf{1 j}$ & $2-\mathrm{ClC}_{6} \mathrm{H}_{4}$ & $4-\mathrm{MeC}_{6} \mathrm{H}_{4}$ & 4 & 1.90 & 1205 & 83 \\
$\mathbf{1 k}$ & $2-\left(\mathrm{Et}_{2} \mathrm{~N}_{2}\right) \mathrm{C}_{6} \mathrm{H}_{4}$ & $4-\mathrm{MeC}_{6} \mathrm{H}_{4}$ & 3 & 1.95 & 1015 & 81 \\
$\mathbf{1 1}$ & $2,4-\left(\mathrm{NO}_{2}\right)_{2} \mathrm{C}_{6} \mathrm{H}_{3}$ & $4-\mathrm{MeC}_{6} \mathrm{H}_{4}$ & 4 & 2.05 & 1184 & 74 \\
\hline
\end{tabular}


Table 2. Antibacterial activity of compounds (1a-11).

\begin{tabular}{|c|c|c|c|c|c|c|c|c|c|c|c|c|}
\hline \multirow[t]{3}{*}{ Compound } & \multicolumn{12}{|c|}{ Zone of inhibition (mm) } \\
\hline & \multicolumn{3}{|c|}{$\begin{array}{c}\text { E. coli } \\
\left(\mu \operatorname{gdisc}^{-1}\right)\end{array}$} & \multicolumn{3}{|c|}{ K. pneumoniae $\left(\mu \mathrm{gdisc}^{-1}\right)$} & \multicolumn{3}{|c|}{$\begin{array}{c}\text { B. subtilis. } \\
\left(\mu \operatorname{gdisc}^{-1}\right)\end{array}$} & \multicolumn{3}{|c|}{$\begin{array}{l}\text { S. aureus } \\
\left(\mu \operatorname{gdisc}^{-1}\right)\end{array}$} \\
\hline & 25 & 50 & 100 & 25 & 50 & 100 & 25 & 50 & 100 & 25 & 50 & 100 \\
\hline 1a & 2 & 6 & 13 & 2 & 5 & 10 & 2 & 8 & 12 & 2 & 6 & 12 \\
\hline 1b & 0 & 4 & 7 & 0 & 3 & 8 & 0 & 3 & 7 & 0 & 2 & 6 \\
\hline 1c & 0 & 5 & 10 & 2 & 6 & 10 & 2 & 7 & 11 & 0 & 6 & 11 \\
\hline 1d & 0 & 7 & 12 & 2 & 6 & 12 & 0 & 7 & 12 & 2 & 6 & 12 \\
\hline 1e & 0 & 2 & 4 & 0 & 0 & 5 & 0 & 2 & 4 & 0 & 2 & 5 \\
\hline 1f & 0 & 5 & 9 & 0 & 4 & 8 & 2 & 4 & 7 & 1 & 4 & 8 \\
\hline $1 \mathrm{~g}$ & 2 & 7 & 14 & 0 & 6 & 11 & 0 & 7 & 11 & 0 & 6 & 12 \\
\hline 1h & 0 & 6 & 12 & 2 & 7 & 10 & 0 & 6 & 10 & 0 & 5 & 11 \\
\hline $1 \mathrm{i}$ & 0 & 5 & 11 & 0 & 5 & 10 & 0 & 7 & 12 & 0 & 5 & 10 \\
\hline $\mathbf{1 j}$ & 2 & 8 & 13 & 0 & 7 & 11 & 2 & 7 & 13 & 0 & 6 & 11 \\
\hline $1 \mathrm{k}$ & 0 & 2 & 5 & 0 & 0 & 4 & 0 & 3 & 5 & 0 & 2 & 4 \\
\hline 11 & 0 & 7 & 11 & 0 & 6 & 11 & 0 & 6 & 12 & 0 & 6 & 10 \\
\hline Penicillin & 0 & 8 & 12 & 2 & 7 & 10 & 2 & 8 & 12 & 2 & 7 & 11 \\
\hline
\end{tabular}

Table 3. Antifungal activity of the compounds (1a-11).

\begin{tabular}{|c|c|c|c|c|c|c|c|c|c|c|c|c|}
\hline \multirow[t]{3}{*}{ Compound } & \multicolumn{12}{|c|}{ Zone of inhibition (mm) } \\
\hline & \multicolumn{3}{|c|}{$\begin{array}{c}\text { A. niger } \\
\left(\mu \operatorname{gdisc}^{-1}\right)\end{array}$} & \multicolumn{3}{|c|}{ P. solmanicolor $\left(\mu \mathrm{gdisc}^{-1}\right)$} & \multicolumn{3}{|c|}{ C. pannical, $\quad\left(\mu \operatorname{gdisc}^{-1}\right)$} & \multicolumn{3}{|c|}{$\begin{array}{l}\text { C. albicans } \\
\left(\mu \operatorname{gdisc}^{-1}\right)\end{array}$} \\
\hline & 25 & 50 & 100 & 25 & 50 & 100 & 25 & 50 & 100 & 25 & 50 & 100 \\
\hline 1a & 2 & 6 & 12 & 0 & 5 & 11 & 2 & 7 & 13 & 2 & 8 & 12 \\
\hline 1b & 0 & 2 & 6 & 0 & 0 & 6 & 0 & 0 & 5 & 0 & 2 & 6 \\
\hline $1 \mathrm{c}$ & 0 & 6 & 10 & 0 & 5 & 10 & 0 & 6 & 11 & 2 & 6 & 11 \\
\hline 1d & 0 & 7 & 13 & 0 & 5 & 10 & 0 & 5 & 10 & 2 & 8 & 12 \\
\hline 1e & 0 & 2 & 7 & 0 & 0 & 5 & 0 & 3 & 6 & 0 & 2 & 7 \\
\hline 1f & 2 & 4 & 7 & 0 & 5 & 8 & 1 & 3 & 6 & 2 & 4 & 6 \\
\hline $1 \mathrm{~g}$ & 0 & 7 & 12 & 0 & 6 & 11 & 2 & 7 & 13 & 0 & 6 & 12 \\
\hline $1 \mathrm{~h}$ & 0 & 5 & 10 & 0 & 5 & 9 & 0 & 6 & 11 & 0 & 5 & 10 \\
\hline $1 \mathbf{i}$ & 0 & 6 & 11 & 0 & 6 & 10 & 0 & 7 & 11 & 0 & 5 & 10 \\
\hline $\mathbf{1 j}$ & 2 & 7 & 13 & 0 & 6 & 11 & 2 & 7 & 12 & 0 & 6 & 11 \\
\hline $1 \mathrm{k}$ & 0 & 5 & 9 & 0 & 4 & 8 & 0 & 3 & 9 & 0 & 6 & 10 \\
\hline 11 & 0 & 6 & 12 & 0 & 5 & 9 & 0 & 6 & 11 & 0 & 5 & 10 \\
\hline Griseofulvins & 0 & 8 & 12 & 2 & 7 & 10 & 2 & 8 & 12 & 2 & 7 & 11 \\
\hline
\end{tabular}

The compounds (1a-11) were screened for their antifungal activity against Aspergillus niger, Crysosporium pannical, Pellicularia solmanicolor and Candida albicans species along with the standard fungicide Griseofulvins 1a-1l (Table 3). The disc diffusion method was followed for screening the compounds at three concentrations $(25,50$ and 100 ppm). The screening results showed that all the compounds displayed good antifungal activity. However, compounds 1a, 1d, $\mathbf{1 g}$ and $\mathbf{1 j}$ showed approximately equal or greater antifungal activity for different strains when compared with the griseofulvin. 
The antimicrobial activity of the compounds varied upon the type and position of the substituents at 5-substituted-2-amino-1,3,4-oxadiazole moiety. It can be concluded from the antimicrobial screening results that when 5-substituted-2-amino-1,3,4oxadiazoles were substituted with aryl halide the antimicrobial activity was altered to an appreciable extent.

\section{ACKNOWLEDGEMENTS}

The author thanks to Sophisticated Analytical Instrument Facility (SAIF) a division of CDRI (Central Drug Research Institute) Lucknow for recording spectra and microanalyses, and The Principal, Iswar Saran Degree College, Allahabad for providing necessary facilities.

\section{REFERENCES}

1. El-Emam, A.A., Al-Deeb, O.A. and Al-Omar, M. 2004. Synthesis, antibacterial and anti-HIV activity of certain 5-(1adamantyl)-2-substituted thio-1,3,4-oxadiazoles and 5-(1adamantyl)-3-substituted aminomethyl-1,3,4-oxadiazolin-2thiones. Bioorg. Med. Chem. 12, 5107-5113.

2. Holla B.S., Gonaslaves R. and Shenoy S. 2000. Synthesis and antibacterial studies of a new series of 1,2-bis(1,3,4oxadiazol-2-yl)ethanes and 1,2-bis(4-amino-1,2,4-triazol-3yl)ethanes. Eur. J. Med. Chem. 35, 267-271.

3. Maslat, A.O., Abussaud, M., Tashtoush, H. and Al-Talib, M. 2002. Synthesis, antibacterial, antifungal and genotoxic activity of bis-1,3,4-oxadiazole derivatives. Pol. J. Pharmacol. 54, 55-59.

4. Xiajuan Z., Zhang Z. and Jin G. 2002. Synthesis and biological activity of 1,3,4-oxadiazole-substituted pyridazinones. J. Chem. Res. 5, 228-230.

5. Zou X.-J., Lai L.-H., Jin G.-Y. and Zhang Z.-X. 2002. Synthesis, fungicidal activity, and 3D-QSAR of pyridazinone-substituted 1,3,4-Oxadiazoles and 1,3,4Thiadiazoles. J. Agric. Food. Chem. 50, 3757-3760.

6 Kucukguzel, S.G., Oruc, E.E., Rollas, S., Sahin, F. and Ozbek, A. 2002. Synthesis, characterization and biological activity of novel 4-thiazolidinones, 1,3,4-oxadiazoles and some related compounds. Eur. J. Med. Chem. 37, 197-206.

7. Chauhan, D., Chauhan, J.S., Singh, J., Bajpai, S.K. and Joshi, M.N. 2003. Synthesis and bioevaluation of some novel nucleosides as antiherptic agents. Indian J. Chem. 42B, 215218 .
8. Kagthara, P.R., Shah, N.S., Doshi, R.K. and Parekh, H.H. 1999. Synthesis of 2,5-disubstituted-1,3,4-oxadiazoles as biologically active heterocycles. Indian J. Chem. 38B, 572576.

9. Mohan, T.P., Vishalakshi, B., Bhat, K.S. and Kendappa, G.N. 2004. Synthesis and insecticidal activity of some 1,3,4oxadiazole derivatives containing phenoxy fluoro group. Indian J. Chem. 43B, 1798-1801.

10. Kennedy, D.A. and Summers, L.A. 1981. Chemical constitution and activity of Herbicides. Part XIV. Reduction potential and herbicidal activity of 4,4-(1,3,4-thiadiazoly-2,5diyl) and 4,4-(1,3,4-oxadiazol-2,5-diyl) bis (1methylpyridinium)diiodides. J. Heterocycl. Chem. 18, 401410.

11. Santagati, M., Modica, M., Santagati, A., Russo, F., Caruso, A. and Cutuli, V. 1994. Synthesis and pharmacological properties of benzothiazole, 1,3,4-oxadiazole and 1,2,4thiadiazole derivatives. Pharmazie 49, 880-884.

12. Mullican, M.D., Wilsonj, M.W., Connor, D.T., Kostlan, C.R., Schrier, D.J. and Dyer, R.D. 1993. Design of 5-(3,5-Di-terbutyl-4-hydroxyphenyl)-1,3,4-thiadiazol-1-yl-1,3,4-oxadia-

zoles and 1,2,4-triazoles as orally active, non ulcerogenic anti-inflammatory agents. J. Med. Chem. 36, 1090-1099.

13. Yale, H.I. and Losee, K. 1966. 2-Amino-5-substituted-1,3,4oxadiazoles and 5-iminosubstituted-2-1,3,4-oxadiazolines: A group of novel muscle relaxants. J. Med. Chem. 9, 478-483.

14. Clapp, L. B., Katritzky, A. R. and Rees, C. W. 1984.Comprehensive Heterocyclic Chemistry, Pergamon Press, Oxford, p. 365.

15. Khan, M.S., Khan, R.M. and Drabu, S. 2001. Anticonvulsant and antibacterial activity of some new 1,3,4-oxadiazole derivatives. Indian J. Heterocycl. Chem. 11, 119-122.

16. Maillard, J., Vincent, M., Morin, R. and Bernard, M. 1962. Hypnotic and sedative drug, 2-(o-hydroxyphenyl)-1,3,4oxadiazole: French Pat M379: Chem. Abstr. 57, 15251g.

17. Jessen, K.A., English, N.M., Wang, J.Y., Maliartenou, S.K., Archer, S.P. and Qiu, L. 2005. The discovery and mechanism of action of novel tumour-selective and apoptosis-inducing 3,5-diaryl-1,2,4-oxadiazole series using a chemical genetics approach. Mol. Cancer Ther. 4, 761-771.

18. Farghaly, A.A., Bekhit, A.A. and Park, J.Y. 2000. Design and synthesis of some oxadiazolyl, thiazolidinyl and thiazolyl derivatives of $1 \mathrm{H}$-pyrazole as anti-inflammatory and antimicrobial agents. Arch. Pharm. 333, 53-57.

19. Hetzheim, A. and Moeckel, K. 1967. Recent advances in 1,3,4-oxadiazole chemistry. Adv Heterocyclic Chem. 7, 183224. 
20. Golovlyova, S.M., Moskvichev, Y.A., Alov, E.M., Kobylinsky, D.B. and Ermolaeva, V.V. 2001. Synthesis of novel five membered nitrogen containing heterocyclic compounds from derivatives of arylsulfonyl and arylthioaceticand propionic acids. Chem. Heterocycl. Compd. 37, 110206 .

21. Hill J. 1984. In: $\mathrm{K} \mathrm{T}$ Potts, Eds., Comprehensive Heterocyclic Chemistry, Pergamon Press, Oxford, 06, 427.

22. Wang, X., Li, Z. and Yang, J. 2002. Synthesis of 2-(4methoxylphenyloxy-acetylamido)-5-aryloxymethyl-1,3,4oxadiazoles under microwave irradiation. Synth. Commun. 32, 1097-1103.

23. Cappo, F.T., Evans, K.A., Graybill, T.L. and Burton, G. 2004. Efficient one pot preparation of 5-substituted-2-amino1,3,4- oxadiazoles using resin bound reagents. Tetrahedron Lett. 45, 3257-3260.

24. Mann, C.K. and Barnes, K.K. 1970. In: Electrochemical Reactions In Non aqueous Systems, Marcel Dekker, Inc. New York, Chapter 1, pp. 13-18.

25. Fry, A.J. 1989. In: Synthetic Organic Electrochemistry, Wiley-Interscience Publication, New York, Chapter 3, pp. 71-78.
26. Shono, T. 1991. In: Electroorganic Synthesis, Academic Press Ltd., London, Chapter 4, pp. 11-19.

27. Sharma, L.K., Kumar, S., Yadav, P. and Singh, R.K.P. 2008. Electrochemical nuclear acetamidation of aromatic compounds at the platinum anode. Indian J. Chem. 47B, 1277-1280.

28. Kumar, S., Sharma, L.K. and Singh, R.K.P. 2006. Electroinduced aldol condensation at platinum electrode. $J$. Indian Chem. Soc., 83, 1160-1162.

29. Kumar, S. 2010. Studies of biologically active heterocycles: synthesis, characterization and antimicrobial activity of some 5-substitutd-2-amino-1,3,4-oxadiazoles. Dhaka Univ. J. Pharm. Sci. 9, 53-59

30. Benson, H. J. 1990. Microbiological Applications, $5^{\text {th }}$ Eds., W. C. Brown Publications, Boston, MA, USA, p. 134

31. Winter, C.A., Risley, E.A. and Nuss, G.W. 1962. Carrageenan induced oedema in hind paw of the rat as an assay for anti-inflammatory drugs. Proc. Soc. Exp. Biol. Med. 111, 544-547. 